\title{
Enzyme Mediated Encapsulation of Gold Nanoparticles by Polyaniline Nanoshell
}

\author{
R. Sfez ${ }^{\# 1,2}$, E. Natan ${ }^{\# 3}$, Y. Bardavid ${ }^{1}$, M. Ikbal ${ }^{1}$, E. Arbeli $^{4}$, \\ Isaiah T. Arkin ${ }^{5}$, I. Popov ${ }^{6}$ and Shlomo Yitzchaik ${ }^{1,6 *}$ \\ ${ }^{1}$ Institute of Chemistry, The Hebrew University of Jerusalem, Jerusalem \\ 91904, Israel \\ ${ }^{2}$ Azrieli, College of Engineering, Jerusalem, Israel \\ ${ }^{3}$ Department of Chemistry, University of Oxford, Oxford OX1 3TA, UK \\ ${ }^{4}$ Department of Chemistry and the National Institute for Biotechnology in the \\ Negev, Ben-Gurion University of the Negev, Be'er-Sheva 84105, Israel \\ ${ }^{5}$ Department of Biological Chemistry, The Alexander Silberman Institute of Life \\ Sciences, The Hebrew University of Jerusalem, Edmund J. Safra Campus, \\ Jerusalem, 91904, Israel \\ ${ }^{6}$ The HU Center for Nanoscience and Nanotechnology, The Hebrew University of \\ Jerusalem, Jerusalem 91904, Israel \\ \# These authors contributed equally to this work \\ *Corresponding author: sy@cc.huji.ac.il
}

Received 14 January 2015; Accepted April 7 2015;

Publication April 142015

\begin{abstract}
In this contribution we describe the formation of gold nanoparticles (AuNP) and polyaniline (PANI) AuNP-PANI nanocomposite via in situ enzymatic polymerization. The method consists of electrostatic adsorption of anilinium monomers on AuNPs citrate stabilized surface of $50 \mathrm{~nm}$ diameters, followed by oxidation with horseradish peroxidase (HRP) enzyme and its cofactor $\mathrm{H}_{2} \mathrm{O}_{2}$. All reaction steps were monitored by UV-Vis-NIR spectroscopy including in situ detection of the polymerization process. UV-Vis-NIR, Cyclic voltammetry (CV) and surface enhanced Raman scattering (SERS) measurements supported the formation of a nanoshell of PANI on the AuNP core.
\end{abstract}

Journal of Self-Assembly and Molecular Electronics, Vol. 3, 1-16.

doi: 10.13052/jsame2245-4551.311

(c) 2015 River Publishers. All rights reserved. 
Two templates for anilinium assembly were compared revealing a strong dependence of the enzymatic kinetics on the template. The kinetic study had shown that the rigid template of the AuNP contributes to higher reaction rate on the AuNP compared with the more flexible polyanion template. The mild reaction condition enables an easy and precise method for obtaining PANI nano-shell on anionic templates for advanced bioelectronic applications.

Keywords: Au-nanoparticles (AuNP), enzymatic polymerization, HRP enzyme, polyaniline, nano-shell.

\section{Introduction}

Gold nanoparticles (AuNP) among other noble metal nanoparticles have attracted considerable attention due to possible applications in numerous fields, such as catalysis, electronics, optics [1] and much more [2]. Conjugated polymers, also known as intrinsically conductive polymers, are widely used in various applications due to their ease of synthesis, low cost and facile control of their optical and electronic properties [3]. Therefore, it is to be expected that hybrid materials composed of both metal NPs and conducting polymers would have a wide range of applications and interesting properties. Indeed, those hybrids which were recently reviewed [4] are already used among other applications for biological [5], electronic [6], catalysis [7] and devices [8]. Polyaniline (PANI) is one of the most used conducting polymers since its discovery a few decades ago because its conductivity depend on both its redox state and proton doping, i.e. the $\mathrm{pH}$ of the environment [9]. The use of anionic templates for adsorption of positively charged monomers followed by in situ chemical, electrochemical and enzymatic polymerization, yielding surface confined nanolayers of conducting polymers was already exemplified for various substrates, such as: metal [10] and oxide surfaces [11], porous silicon [12] carbon nanotubes [13] and DNA [14]. These experiments are setting the stage for the use of new in situ template synthesis on negatively charged AuNPs.

In this work we have chosen to focus on a core shell hybrids composed of a $50 \mathrm{~nm}$ AuNP core, covered by a monolayer shell of in situ enzymatically polymerized PANI. This specific hybrid might be of interest in various applications, like in memristive devices [15a] and as a good template for neuron growth $[15 \mathrm{~b}]$. The synthetic method consists of electrostatic adsorption of positively charged anilinium monomers on the negatively charged NP surface followed, by oxidation with horseradish peroxidase (HRP) 
enzyme and its cofactor $\mathrm{H}_{2} \mathrm{O}_{2}$. Typically, in order to build nanocomposites of Au core and PANI shell many methods have been used, among which, the use of conducting polymers as reductants [4] or the use of the anilinium monomer as a reductant of the metal salt while oxidative polymerization takes place [16]. Of course, electrochemical methods are widely used also [17]. In general, it is very difficult to obtain a controlled monolayer nanoshell on NP mainly due to aggregation problems during the coating process or growth pattern which is dictated by the organic component. Some attempts were conducted on AuNP-PEDOT [18] or Au-polypyrrole hybrids [19], and also with polyaniline using chemical oxidation [20]. In our work, we wanted to build a controlled nano-shell of PANI on Au-NP core by in situ enzymatic polymerization which afford moderate $\mathrm{pH}$ environment ( $\mathrm{pH} 4.3$ ), and control over the reaction conditions and product by governing the thickness of the formed PANI monolayer. The motivation for this research arises from the need to clarify fundamental aspects of enzymatic polymerization at nanometric confined geometries and characterization of the obtained nano-composite. Enzymatic polymerization of anilinium monomers electrostatically attached to poly (4-styrenesulfonate) (PSS) using HRP was described few years ago [21] and extensively used since then. The use of enzymes as catalysts in chemistry is wide spread [22] due to their specificity and their ability to afford reactions' control by using inhibitors, temperature and $\mathrm{pH}$ modulation which alter their reactivity dramatically. HRP is comprehensively used for redox reactions including anilinium polymerization, allowing a relatively high $\mathrm{pH}$ environment (up to 4.3) compare the typical $\mathrm{pH}$ environment which is much lower (1-2 for electrochemical and chemical oxidations). Following previous works [23] which showed the activity of HRP enzyme on various templates including 2D modified surfaces [24], our goal being to try and elucidate the role of the template on the action and kinetics of the enzymatic reaction while obtaining the desired nanoshell of PANI around Au-NP core. Two templates were compared for polymerizing anilinium monomers: the polyanion PSS and $50 \mathrm{~nm}$ Au-NP. These templates differ dramatically in their rigidity. Three experiments were conducted in which systems without any template, with $50 \mathrm{~nm} \mathrm{Au-NP}$ template and with PSS linear polyanion template were compared. In the template containing experiments, anilinium was electrostatically adsorbed to the template surface (by sulfonate groups in the case of PSS or by carboxylate groups in the case of the citrate capped $50 \mathrm{~nm}$ Au-NP). Enzymatic polymerization took place after proper dialysis process which eliminates polymerization of unabsorbed monomers. It had already been proved that the most important role of the polymeric template 
in enzymatic polymerization is to provide the preferential orientation of the anilinium in order to get the head to tail polymerization [25]. We have find by comparing the different templates that the rigidity parameter is important from kinetics point of view, as could be expected. Indeed it was already recently reviewed [26], that assembling enzymes or substrates to metal nanoparticles has an important influence on the kinetics of the system. All reaction steps were monitored by UV-Vis-NIR spectroscopy including in situ detection of the polymerization process. HR-TEM analysis gave morphological analysis of the monomeric and polymeric layer on the Au-NP. Surface enhanced Raman scattering (SERS) analysis was conducted in order to compare the monomeric form and polymeric form of the nanolayer coating the NP. Cyclic voltammetry (CV) measurements were conducted on PANI coated AuNP attached to modified ITO surfaces, acting as a working electrode, and gave evidence for the presence of the PANI layer. The kinetic role comparison of the different templates was conducted by UV-Vis-NIR spectroscopy using time course mode. The kinetic study had shown that the rigid template of the AuNP contributes to higher reaction rate on the Au-NP compared with the more flexible polymeric template with the same surface number density of anionic (sulfonate) groups.

\section{Experimental Details}

\subsection{Chemicals}

$\mathrm{HAuCl}_{4}, \mathrm{PSS}\left(\mathrm{M}_{\mathrm{W}}=70,000\right) \mathrm{HRP}$ (Aldrich), were used as received. Aniline (Aldrich) was vacuum distilled before use.

\subsection{Synthesis}

Gold nanoparticles capped with citrate ligands were synthesized as was described elsewhere [27]. Briefly, a solution of $0.01 \%$ of $\mathrm{HAuCl}_{4}$ was mixed and heated with changing volumes of $1 \%$ wt. of sodium citrate solution, resulting in the formation of AuNP capped by citrate ligands.

The in situ enzymatic polymerization process took place on the anilinium covered Au-NP. In order to obtain this composite, anilinium solution $(50 \mathrm{mM}$, $\mathrm{pH}$ 4.0) was added drop wise to synthetized citrate covered Au-NP followed by a $30 \mathrm{~min}$ incubation in an ice bath $\left(4^{\circ} \mathrm{C}\right)$ (Scheme 1, step 1). Following previous works which describes aggregation of anilinium [28] a thorough dialysis process was included. Dialysis was carried out against a volume of 


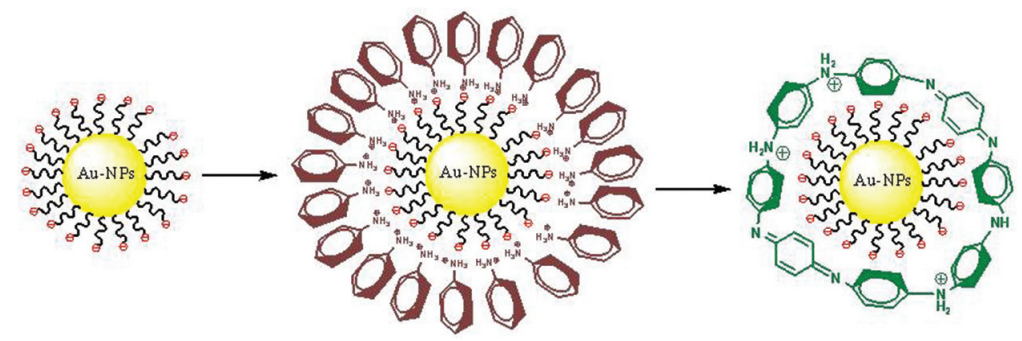

Scheme 1 Formation mechanism of Au-NP-PANI nanocomposite via enzymatic polymerization. Step 1: electrostatic adsorption of positively charged anilinium monomers to negatively charged NP. Step 2: enzymatic polymerization of anilinium nanoshell converted to PANI nanoshell

1 litter $\mathrm{HCl}(\mathrm{pH} 4.0)$ solution, three times for 15 minutes, in order to eliminate unadsorbed anilinium monomers and avoid their bulk polymerization.

The anilinium covered Au-NP was then let to react with $\operatorname{HRP}(1.2 \mu \mathrm{g} / \mathrm{ml}$, room temprature) and $\mathrm{H}_{2} \mathrm{O}_{2}(1.2 \mathrm{nM}$, added drop wise) solutions (scheme 1, step 2) for different periods of time, ranging from 10-90 min. The product was characterized by HR-TEM, UV-Vis and SERS analysis. All reactions were monitored by in situ UV-Vis-NIR spectroscopy. While using PSS as a template for kinetics study a known procedure was used [21] with dialysis as mentioned above.

PSS modified ITO substrates for CV analysis were prepared according to a procedure described elsewhere [24]. Briefly, ITO electrodes were modified with APTMS by using 1\% solution of APTMS in methanol followed by washing with methanol and drying with $\mathrm{N}_{2}$. APTMS modified ITO substrate was placed in aqueous solution of PSS for 1 hour and rinsed with water. The negatively charged PSS modified substrate was incubated in PANI coated Au-NP solution overnight allowing electrostatic adhesion of the positively charged core-shell NPs.

\subsection{Instrumentation}

UV-Vis-NIR spectra were acquired with a Shimadzu spectrophotometer UV3101PC. HR-TEM measurements were conducted with a Tecnai F20 G ${ }^{2}$ operating at $200 \mathrm{KeV}$ including Energy Dispersion X-ray Spectroscopy (EDS) analysis of elements. A 400 mesh size carbon coated copper grid was used for the analysis. Samples for SERS were excited in a backscattering geometry with $488 \mathrm{~nm}$ irradiation from an INNOVA 90C FRED Argon-Ion laser (Coherent Inc., Santa Clara, CA, USA). Spectra were 
recorded by a T64000 triple Raman spectrometer (Jobin-Yvon Horiba, Longjumeau, France) equipped with a liquid nitrogen-cooled Spectrum One CCD detection system (Jobin-Yvon Horiba) in the range of $400 \mathrm{~cm}^{-1}$ to $1700 \mathrm{~cm}^{-1}$ with the spectral resolution of $3 \mathrm{~cm}^{-1}$. Raman frequencies were calibrated using the $934 \mathrm{~cm}^{-1}$ band of $\mathrm{NaClO}_{4}$. The reported spectra are the accumulated averages of 25 exposures of $3 \mathrm{sec}$ each. For all measurements, the spectra of $\mathrm{Au}$ nanoparticles in acid solution $(\mathrm{HCl}, \mathrm{pH} 4.0)$ was taken as a background. Electrochemical measurements were carried out by using AUTOLAB PGSTAT12 (Eco Chemie B.V). Modified ITO substrate was used as a working electrode, a platinum wire as a counter electrode, $\mathrm{Ag} / \mathrm{AgCl}$ saturated $\mathrm{KCl}$ as a reference electrode, and $0.1 \mathrm{M} \mathrm{HCl}$ was used as supporting electrolyte.

The cyclic voltammograms $(\mathrm{CV})$ were recorded by applying voltage between $1.0-0.0 \mathrm{~V}$ at $50 \mathrm{mV} / \mathrm{s}$ scan rate.

\section{Results and Discussions}

HR-TEM analysis was conducted on both the anilinium monomeric and PANI polymeric coated NP after a proper dialysis process. The first step consisted with the formation of the monomeric layer (Figure 1a) of anilinium electrostatically bound to the negatively charged NP. The obtained layer is imaged as a thin nanoshell that can be estimated to be of about $2 \mathrm{~nm}$ width after drying. TEM measurements indicated that no aggregation have occurred. Moreover, the characteristic peak of N 1s was observed only on the hybrid but not on the grid as indicated by EDX (Suppl. 1). These results support the claim that no effective concentration of anilinium remained in the solution after the consecutive dialysis. The second step consisted of the in situ enzymatic oxidation and polymer formation. The in situ enzymatic polymerization was conducted on the anilinium free solution containing anilinium covered NP at $\mathrm{pH}$ 4.0. Different time periods were examined and fifteen minutes was determined to be the optimal time in order to get polymerization and avoid aggregation.

A fifteen minutes polymerization time resulted in the formation of a thin layer of the polymer (Figure 1b). Almost no change in the nanoshell width was observed upon increasing polymerization time, supporting our assumption that no free anilinium monomers remained in the solution after dialysis. However, upon polymerization more hybrids were observed as pairs or small aggregates. This fact can be explained by the polymerization which takes place on one particle and continues on the nearest particle where anilinium monomers could 

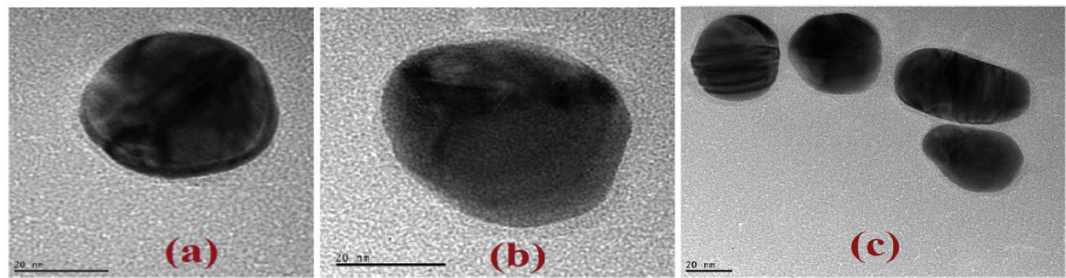

Figure 1 HR-TEM images of (a) $50 \mathrm{~nm}$ Au-NP coated by anilinium monomer nanoshell. (b) PANI nanoshell after 15 min of in situ enzymatic polymerization. (c) same as (b) for a group of NPs after polymerization (Scale bar $20 \mathrm{~nm}$ )

be still polymerized (Figure 1c). While letting a longer reaction time we got different polymeric matrices embedded Au-NP aligning the NP was observed. It is important to notice that no trace of the enzyme was observed in the final product. While replacing the HRP enzyme by a mutant HRP lacking of active site no polymerization was detected, as was already proved for surfaces [24].

UV-Vis-NIR analysis was conducted on the monomer covered NP giving rise to the Au surface plasmon at $537 \mathrm{~nm}$ as expected by the size of the NP (Figure 2), indicating that no aggregation had occurred (see Supple. 2, for detailed UV-Vis). The enzymatically oxidized anilinium-monomer coated NP gave rise to a broad spectra indicating PANI formation (Figure 2), and

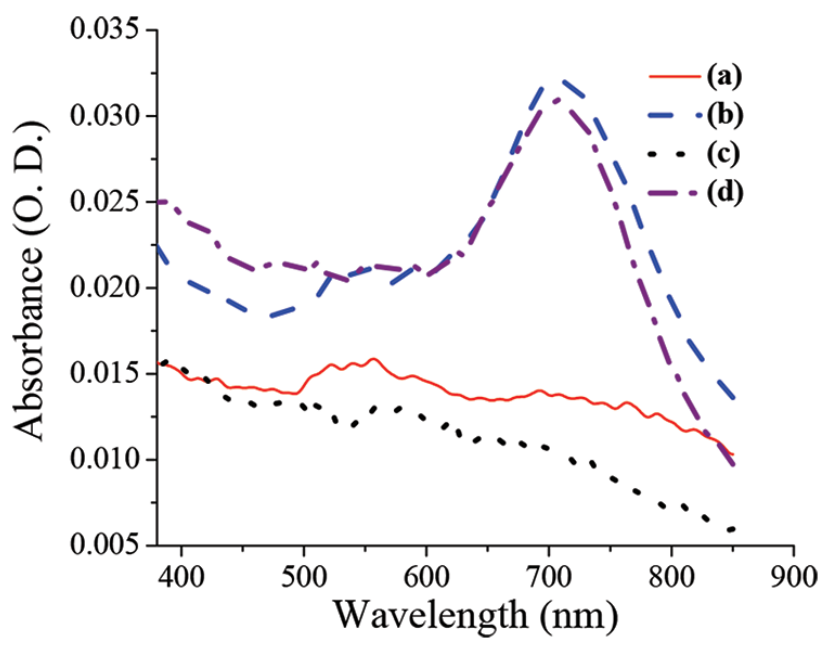

Figure 2 Comparison of UV-Vis.-NIR spectra of PANI polymer obtained on the different templates: (a) anilinium coated Au-NP showing characteristic plasmon at $537 \mathrm{~nm}$, (b) PANI coated Au-NP (30 min), (c) anilinium coated PSS, and (d) PANI coated PSS (30 min) 
red shift of the surface plasmon, probably due to polymer formation. While characterizing the PSS template for polymerization, UV-Vis-NIR analysis has shown clearly that the obtained polymer on both templates is the same (Figure 2).

The only difference between the spectra is the Au surface plasmon which appears in the Au-PANI nanocomposite, but lack on the PSS, as expected. SERS analysis was conducted on samples with higher concentration than was needed for TEM and UV-Vis. While comparing the SERS analysis obtained for the monomer and the polymer, two main features were observed (Figure 3). The first one is the broadening of the peaks for the polymer comparing the monomer (sharp peak at $1200 \mathrm{~cm}^{-1}$ for the monomer comparing broad peak from $1100 \mathrm{~cm}^{-1}$ to $1350 \mathrm{~cm}^{-1}$ for the polymer) suggesting oligomer or polymer formation. The second feature is the newly appearing peaks characteristic of quinoid group (such as $\mathrm{C}-\mathrm{C}$ bond in quinoid ring at $1580 \mathrm{~cm}^{-1}$ or C-H bending in quinoid ring at $1158 \mathrm{~cm}^{-1}$ ) which exist in the polymer but lack in the monomer, thus supporting polymer formation [29].

$\mathrm{CV}$ measurements were conducted on the modified ITO electrode immersed in $\mathrm{HCl}$ solution $(\mathrm{pH}=4)$. As can be shown (Figure 4) a redox couple appears at $0.30 \mathrm{~V}$ (cathodic) and $0.48 \mathrm{~V}$ (anodic) which was attributed to the

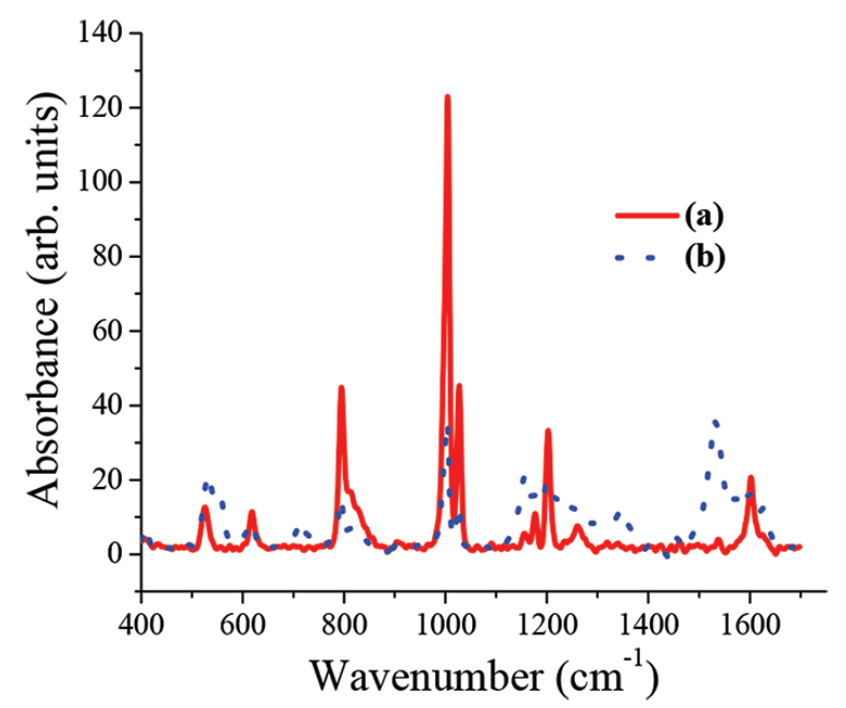

Figure 3 Raman spectra of (a) the anilinium monomer and (b) Polymer adsorbed over the AuNP's, at $\lambda=488 \mathrm{~nm}$. The presented spectra are obtained following background subtraction (AuNP's solution) 


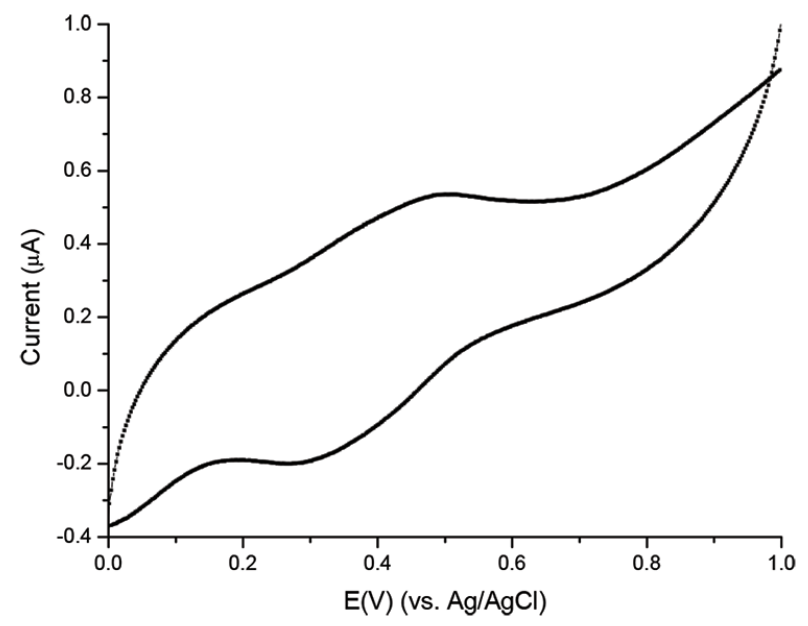

Figure $4 \mathrm{CV}$ of Au-PANI nanocomposites electrostatically adsorbed to PSS modified ITO surface. Modified ITO surface was used as a working electrode, a platinum wire as a counter electrode, $\mathrm{Ag} / \mathrm{AgCl}$ saturated $\mathrm{KCl}$ as a reference electrode, and $0.1 \mathrm{M} \mathrm{HCl}$ was used as supporting electrolyte. Scan rate for the range of $1.0-0.0 \mathrm{~V}$ at $50 \mathrm{mV} / \mathrm{s}$

reduction and oxidation process of the PANI nanoshell, although a bit shifted comparing PANI characteristic values [10, 11a]. These results are similar to PANI polymerized on nano-confined templates [30], and support the formation of PANI nanoshell on Au-NP. Control experiments were conducted with non-coated Au-NP that gave no PANI signal mainly due to the electrostatic repulsion between the PSS modified ITO and the negatively charged particles. The PANI nanolayer obtained therefore is not stable and thus explaining the lack of redox peaks in the voltammogram.

Kinetics study was carried out with time course spectroscopic analysis while choosing the wavelength to be $709 \mathrm{~nm}$. This wavelength was chosen because it is characteristic of PANI's bipolaron absorption, which increases with time.

As can be seen (Figure 5), the control experiment which was conducted without a template shows no significant change with time. However, comparison of the two templates, i.e. Au-NP and PSS, show an increase in the peak intensity but with different growth rates.

The rate of PANI growth on the rigid NP is higher than the growth on the more flexible linear PSS by a factor of two $\left(k_{o b s .}^{A u}=\right.$ $3.95 \cdot 10^{-5} \mathrm{sec}^{-1}$ vs. $\left.k_{\text {obs. }}^{P S S}=2.06 \cdot 10^{-5} \mathrm{sec}^{-1}\right)$. This difference in rigidity can account for the different enzymatic rate of polymerization. The mechanism 


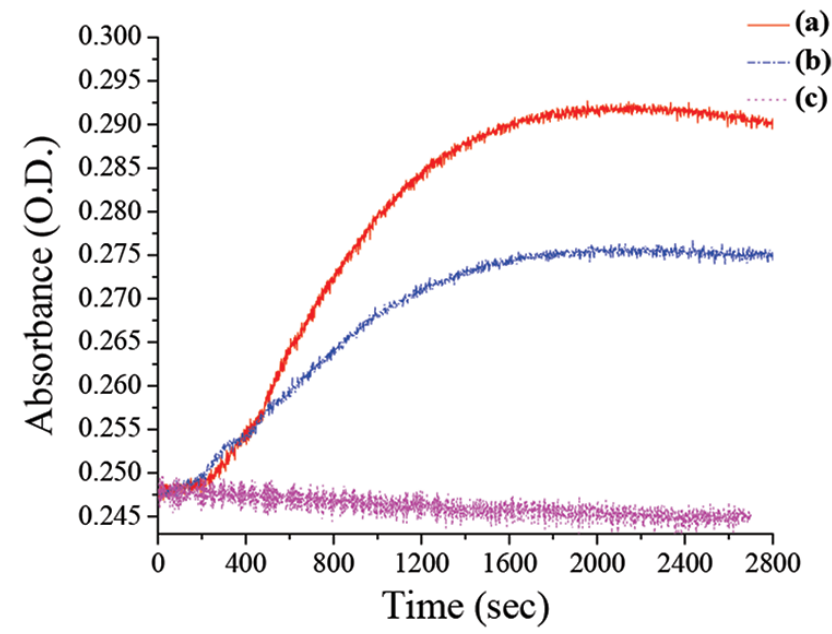

Figure 5 UV-vis. driven kinetic experiment tracing $709 \mathrm{~nm}$ with time for different experimental conditions of enzymatic polymerization: (a) the AuNP's template (b) PSS template (c) no template

of the enzyme action remains unclear; however we think that we can consider the action like the action on a $2 \mathrm{D}$ surface due to the fact that the enzyme active site is about $20 \AA^{3}$, and so the NP which has a diameter of $50 \mathrm{~nm}$ can be considered as a "flat" surface. Comparison of chemical and enzymatic oxidation shows the advantages of the enzymatic polymerization with regard to the condition of the polymerization and the control over the reaction and the obtained aggregation free PANI encapsulated Au-NP.

\section{Summary}

We had shown a way to get nano-composites of Au-NP-PANI by in situ enzymatic polymerization achieved in facile and moderate conditions, using $\mathrm{HRP}$ in the presence of $\mathrm{H}_{2} \mathrm{O}_{2}$. Structural characterization showed clearly a nanolayer formation of in situ enzymatically polymerized PANI. CV, UVVis-NIR and SERS measurements supported the formation of a nano-shell of PANI on the Au-NP. It is a first example of in situ enzymatic polymerization of anilinium monomers to PANI on Au-NP. The role of the template was studied kinetically and had shown a dependence of the enzymatic reaction rate on the rigidity of the template. This moderate method can be used in the future as a tool for gentle synthesis with various NP's and many horizons in bionic and nanoelectronic driven devices. 


\section{Acknowledgements}

This work is supported by the FP7 318597 SYMONE project (FET-UCOMP thematic area) and in part by COST action MP1202 (HINT).

\section{References}

[1] G. Baffou, R. Quidant. Chem. Soc. Rev., 43, 3898-3907 (2014).

[2] a) S. Yang, X. Luo. Nanoscale, 6, 4438-4457, (2014); b) M. C. Daniel, D. Astruc. Chem. Rev. 104, 293-346 (2004).

[3] J. Pecher, S. Mecking. Chem. Rev., 110, 6260-6279 (2010).

[4] P. Xu, X. Han, B. Zhang, Y. Du, H. L. Wang. Chem. Soc. Rev., 43, 1349-1360 (2014).

[5] M. Strivastava, S. K. Strivastava, N. R. Nirala, R. Prakash.Anal. Methods, 6, 817-824, (2014).

[6] S. Huh, S. B. Kim. J. Phys. Chem. C, 114, 2880-2885 (2010).

[7] X. Liu, L. Li, M. Ye, Y. Xue, S. Chen. Nanoscale, 6, 5223-5229 (2014).

[8] Y. Leroux, E. Eang, C, Fave, G. Trippe, J. C. Lacroix. Electrochem. Comm. 9, 1258-1262, (2007).

[9] a) A. J. Heeger. J. Phys. Chem. B, 105, 8475-8491 (2001); b) E. Smela. Adv. Mat. 15, 481-494 (2003); c) J. C. Chiang, A. G. MacDiarmid. Synth. Met. 13, 193-205 (1986); d) E. W. Paul, A. J. Rico, M. S. Wrighton. J. Phys. Chem. 89, 1441-1447 (1985).

[10] I. Turyan, D. Mandler. J. Am. Chem. Soc. 120, 10733-10742 (1998).

[11] a) R. Sfez, L. De-Zhong, I. Turyan, D. Mandler, S. Yitzchaik. Langmuir, 17, 2556-2559 (2001); b) R. Oren, R. Sfez, N. Korbakov, K. Shabtai, A. Cohen, H. Erez, A. Dormann, H. Cohen, J. Shappir, M.E. Spira, S. Yitzchaik. J. Biomater. Sci. Polymer Edn, 15, 1355-1374 (2004).

[12] A. Nahor, I. Shalev, A. Sa'ar, S. Yitzchaik. Eur. J. Inorg. Chem., 2014, in press. DOI: 10.1002/ejic.201402450.

[13] a) S. Ben-Valid, H. Dumortier, R. Sfez, M. Décossas, A. Bianco, S. Yitzchaik. J. Mater. Chem., 20, 2408-2417 (2010); b) S. Ben-Valid, B. Botka, K. Kamarás, A. Zeng, S. Yitzchaik. Carbon, 48, 2773-2781 (2010).

[14] Y. Bardavid, A. B. Kotlyar, S. Yitzchaik. Macromol. Symp., 2006, 240, 102-106.

[15] a) F. Alibart, S. Pleutin, O. Bichler, C. Gamrat, T. Serrano-Gotarredona, B. Linares-Barranco, D. Vuillaume. Adv. Funct. Mater. 22, 609-616 (2012). b) R. Oren, R. Sfez, N. Korbakov, K. Shabtai, A. Cohen, 
12 R. Sfez et al.

H. Erez, A. Dormann, H. Cohen, J. Shappir, M. E. Spira, S. Yitzchaik. J. Biomater. Sci. Polymer Edn, 15, 1355-1374 (2004).

[16] a) Y. C. Liu. Langmuir, 18, 9513-9518 (2002); b) X. Dai, Y. Tan, J. Xu. Langmuir, 18, 9010-9016 (2002); b) T. K. Sarma, D. Chowdhury, A. Paul, A. Chattopadhyay. Chem Comm. 1048-1049 (2002).

[17] Z. Li, Y. Li, W. Lin, F. Zheng, J. Laven. Polymer Composite, DOI $10.1002 / \mathrm{pc}$.

[18] P. Xu, K. Chang, Y. Il Park, B. Zhang, L. Kang, Y. Du, R. S. Iyer, H. L. Wang. Polymer, 54, 485-489 (2013).

[19] T. Selvan, J. P. Spatz, H. A. Klok, M. Möller. Adv. Mater., 10, 132-134 (1998).

[20] a) K. Mallick, M. J. Witcomb, M. S. Scurrel. Gold Bulletein, 39, 166-174 (2006); b) X. Xu, X. Liu, Q. Yu, W. Wang, S. Xing. Colloid. Polym. Sci., 290, 1759-1764 (2012).

[21] L. A. Samuelson, A. Anagnostopolulos, K. S. Alva, J. Kumar, S. K. Tripathy. Macromolecules, 31, 4376-4378 (1998).

[22] K. M. Koeller, C. Wong. Nature, 409, 232-240 (2001).

[23] W. Liu, J. Kumar, S. K. Tripathy, K. J. Senecal, L. A. Samuelson. J. Am. Chem. Soc. 121, 71-78 (1999).

[24] R. Sfez, N. Peor, S. R. Cohen, H. Cohen, S. Yitzchaik. J. Mater. Chem. 16, 4044-4050 (2006).

[25] W. Liu, A. L. Cholli, R. Nagarajan, J. Kumar, S. Tripathy, F. F. Bruno, L. Samuelson. J. Am. Chem. Soc. 121, 11345-11355 (1999).

[26] B. J. Johnson, W. Russ Algar, A. P. Malanoski, M. G. Ancona, I. L. Medintz. Nanotoday, 9, 102-131, (2014).

[27] G. Frens. Nature-Physical Science, 241, 20-22 (1973).

[28] D. S. Janelle Newman, W. A. MacCrehan. Langmuir, 25, 8993-8998, (2009).

[29] a) M. Baibarac, M. Cochet, M. Lapkowski, L. Mihut, S. Lefrant, I. Baltog. Synt. Met. 96, 63-70, (1998); b) M. Lapkowski, K. Berrada, S. Quillard, G. Louarn, S. Lefrant, A. Pron. Macromolecules, 28, 1233-1238 (1995).

[30] K. Kamarás, B. Botka1, A. Pekker1, S. Ben-Valid, A. Zeng, L. Reiss, S. Yitzchaik. Phys. Status Solidi B, 246, 2737-2739 (2009). 


\section{Biographies}

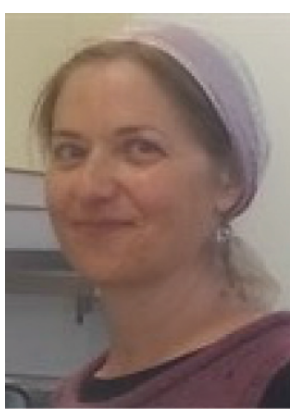

R. Sfez got her Ph.D. in Chemistry at the Hebrew University of Jerusalem, in 2003. She continued as a researcher at the university for several years. Since 2011 she is a senior lecturer at the Advanced materials engineering department and head of academic studies in Chemistry at Azrieli, college of engineering, Jerusalem. Her current research interests are self- assembled monolayers for various applications and nanomaterials hybrids.

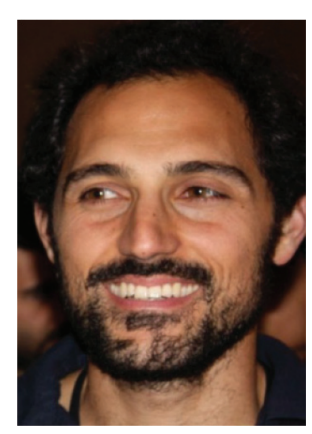

E. Natan received his BSc. in Biology, and later a MSc. in Organic Chemistry under the supervision of Professor Shlomo Yitzchaik, both at the Hebrew University of Jerusalem. He conducted his $\mathrm{PhD}$ in Cambridge University in the lab of Professor Alan R Fersht and joined as a postdoc to the Teichmann lab in the EMBL European Bioinformatics Institute. His research activity is in the field of protein structure, folding and stability. 


\section{R. Sfez et al.}

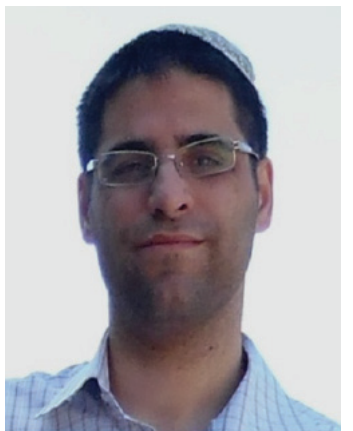

Y. Bardavid was born in Istanbul Turkey,1978. He got his Ph.D. from Hebrew University of Jerusalem in 2010. He is working at Nanonics, an Israeli Nanotechnology company since 2010 as a researcher.

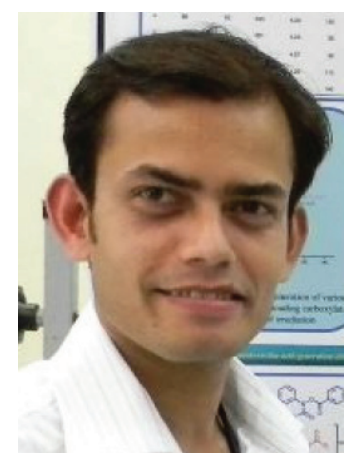

M. Ikbal was born in West Bengal, India, February 1983. In 2005 he got his B. Sc degree in Chemistry from Vidyasagar University, India and from same University he received his M. Sc degree in Organic Chemistry in 2007. He obtained his Ph.D. degree in Organic Photochemistry, under supervision of Prof. N. D. Pradeep Singh, from Indian Institute of Technology Kharagpur. Currently he is a Postdoctoral Fellow in the group of Prof. Shlomo Yitzchaik. His recent research focuses on the design and fabrication of memristive elements based on spiropyran functionalized Au-NPs. 


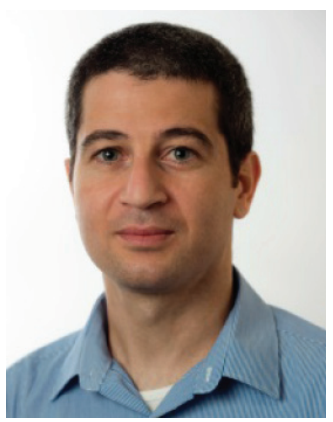

E. Arbely graduated in Chemistry and received his Ph.D. in Biochemistry and Biophysics at the Hebrew University of Jerusalem in 2008. He was a postdoctoral fellow at the MRC Laboratory of Molecular Biology in Cambridge, where he worked under the guidance of Prof. Sir Alan Fersht and Dr. Jason W. Chin. Since 2012 he is a faculty member at the Department of Chemistry, Ben-Gurion University, Israel. His research activity is in the field of synthetic biology, with particular interest in developing and applying methods for genetic code expansion to study the structural and functional role of protein post-translational modifications.

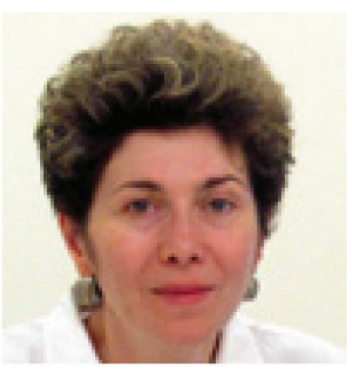

I. Popov graduated in Physical Metallurgy and got her $\mathrm{PhD}$ in Solid State Physics at the Donetsk Physics and Engineering Institute in 1995. At 1997-2001 she was a post-doc fellow at the group of professor Dan Shechtman (Nobel prize in Chemistry 2011) where she studied phase transformations and precipitation of quasi-crystalline phases. From 2001 she is in charge of the Unit for Nanoscopic Characterization and the Hebrew University Center for Nanoscience and Nanotechnology. She is an author of about 70 peer reviewed journal papers. Her research activities are currently focused on development of advanced methods for characterization of materials. 


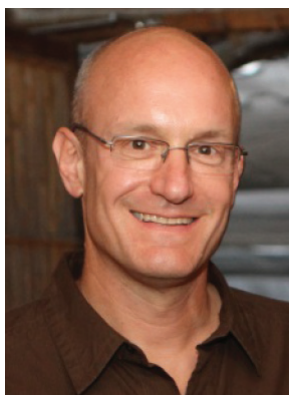

I. (Shy) Arkin studied biology at the Hebrew University of Jerusalem and Tel Aviv University and obtained his $\mathrm{PhD}$ degree from Yale University. His major research interests are membrane protein structure and function, alongside development of tools to investigate them. His group uses experimental approaches, as well as computational tools to analyze the mechanism of membrane transport systems that regulate salinity and acidity of human pathogens. One example is using extensive computational simulations and experimental assays his group elucidated the mechanism of the main $\mathrm{Na}^{+}$ pump of bacteria. Similar efforts in his group uncovered novel mechanisms for drug resistance of the flu virus and potential ways to overcome it. Finally, his group is well known for developing isotope edited FTIR spectroscopy in general, and the ${ }^{13} \mathrm{C}={ }^{18} \mathrm{O}$ probe in particular for structural studies of proteins.

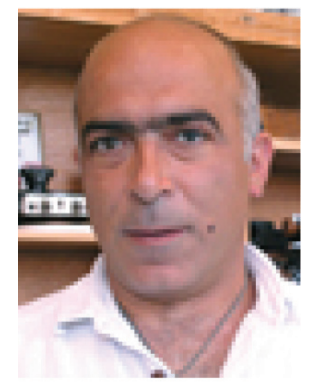

S. Yitzchaik graduated in Chemistry and got his Ph.D. in Chemistry at the Weizmann Institute of Science, Rehovot in 1992. A faculty member of the Hebrew University since 1996. He is an author of about 100 peer reviewed journal papers. Major research interests since 1996 focus around self-assembled nanolayers. He has authored over 100 papers and holds 20 patents in the fields of material science, solid-state organic chemistry and nanolayers derived biosensor technologies. 